\title{
Rapports de voisinage et développement : la responsabilité sans faute selon la Cour suprême du Canada
}

\author{
Jeanne Simard et Marc-André Morency \\ Laboratoire de recherche et d'intervention en gouvernance des organisations (LARIGO) \\ Université du Québec à Chicoutimi
}

\section{INTRODUCTION}

Le jugement de la Cour suprême du Canada dans l'affaire Ciment du Saint-Laurent ne fait que confirmer l'inscription de notre droit et de nos mentalités dans des efforts internationaux visant la qualité de l'environnement et des établissements humains.

En novembre 2008, la Cour suprême du Canada rendait un jugement, dans l'affaire Ciment du Saint-Laurent inc. c. Barrette ${ }^{1}$ (ci-après Ciment du Saint-Laurent). Ce jugement suscite depuis un vif intérêt dans de nombreuses communautés et dans les milieux juridiques, industriels et environnementaux.

À l'issue d'un long processus, un recours collectif, amorcé en 1994 par un groupe représentant des riverains de cette entreprise (alors installée dans l'ancienne ville de Beauport, près de Québec), s'estimant lésés par ses opérations, la Cour a rendu un verdict d'un caractère nouveau en matière de troubles de voisinage sur la base de la responsabilité objective sans faute. Elle a alors accordé un dédommagement substantiel aux plaignants, victimes de troubles anormaux ou excessifs résultant des activités riveraines de la cimenterie.

Des analystes ont qualifié cette décision de «r(évolution) juridique» en raison de ses implications pour les rapports de voisinage et, par ricochet, pour le «développement» industriel et urbain. Pour d'autres, le jugement de la Cour suprême du Canada dans l'affaire Ciment $\mathrm{du}$ Saint-Laurent ne fait que confirmer l'inscription de notre droit et de nos mentalités dans des efforts internationaux visant la qualité de l'environnement et des établissements humains.
Fondée sur des principes énoncés dans le droit civil québécois, cette décision est de nature à intéresser les citoyens de cette province (on est tous le voisin de quelqu'un) et les organisations œuvrant sur une vaste gamme de territoires plus ou moins populeux.

Les gestionnaires d'une organisation, d'une association, d'un syndicat ou d'une entreprise, ne peuvent plus, dans cette province, prétendre écarter toute incertitude dans leur prise de décision, dans leur gestion du risque. Ils doivent désormais avoir conscience que, même s'ils ne commettent aucune faute au sens légal du terme, même s'ils respectent les certificats d'autorisation d'exploitation, leurs actions pourraient néanmoins avoir des conséquences juridiques et économiques majeures. Comme les enjeux environnementaux auront des impacts sur les résultats financiers et la réputation des organisations, des mesures de prévention efficaces et proportionnées de troubles graves doivent être adoptées. On devra procéder à une évaluation plus serrée des risques environnementaux et sociaux au cours des opérations de financement de projets. De plus, les conditions d'assurabilité pourraient faire l'objet d'un examen plus rigoureux.

Comme les enjeux environnementaux auront des impacts sur les résultats financiers et la réputation des organisations, des mesures de prévention efficaces et proportionnées de troubles graves doivent être adoptées. On devra procéder à une évaluation plus serrée des risques environnementaux et sociaux au cours des opérations de financement de projets. 
De fait, dès la publication du jugement, les journaux rapportaient le désir de plusieurs groupes environnementaux et sociaux, à l'instar des opposants au projet méthanier Rabaska à Lauzon, près de Québec, d'entamer des recours collectifs pour faire bloquer ou modifier certains projets susceptibles d'occasionner des inconvénients anormaux «qui excèdent les limites de la tolérance » à la population. La ville de Longueuil tenait, en mars 2010, des audiences publiques sur le bruit émanant de l'aéroport de Saint-Hubert, résultant de la multiplication des écoles de pilotage. On sait que le site est maintenant enclavé dans le tissu urbain. Ces audiences avaient pour but de recueillir les solutions proposées par le milieu et d'évaluer toute proposition pouvant régler le problème. Un procès s'est d'ailleurs ouvert à ce sujet en janvier 2011. Pour sa part, le gouvernement du Québec s'est désisté des procédures d'appel d'un jugement de la Cour supérieure qui le condamnait, en 2004, à verser une indemnité d'un peu plus de 10 millions (\$) à des citoyens de Mont-Tremblant, riverains du parc linéaire Le P'tit Train du Nord, en raison du bruit excessif que leur occasionnait le passage des motoneiges $^{2}$. Enfin, une recherche sur le site de la Société québécoise d'information juridique (SOQUIJ) révèle qu'entre novembre 2008 et juin
2011, la décision de la Cour suprême a été appliquée dans 4 jugements de la Cour supérieure, 4 jugements de la Cour du Québec et mentionnée dans 39 autres décisions de différents tribunaux du Québec.

Dans cet article, nous allons exposer les faits saillants de l'affaire Ciment du St-Laurent et l'argumentation juridique novatrice qu'elle a suscitée. Après une brève présentation du régime de responsabilité fondé sur la faute, qui est le régime de droit commun depuis des années en droit civil québécois, nous montrerons comment s'en distingue le régime de responsabilité sans faute. On percevra mieux alors les implications éthiques de ce nouveau type de responsabilité qui accroît, d'une part, l'incidence de la sphère juridique proprement dite sur la société civile et son économie, mais qui appelle en même temps à une approche participative des problèmes, à la définition de nouveaux contrats moraux. Ce jugement rejoint en somme le vaste mouvement social qui appelle la gouvernance des organisations, des personnes morales, à prendre en considération toutes les parties prenantes dans la prise de décision. Voilà qui illustre bien toute l'étendue de la difficulté de décider en situation complexe.

\section{L'AFFAIRE CIMENT DU SAINT-LAURENT : LES FAITS}

Dès le début de l'exploitation de la Compagnie Ciment du Saint-Laurent, en 1955, des voisins mécontents se plaignent des nuisances résultant des activités de l'usine. Le ministère de l'Environnement intervient à plusieurs reprises dans les années 80, à l'égard de plaintes formulées par les citoyens relativement à des problèmes de poussière, d'odeurs et de bruits importants ou incessants.

La compagnie Ciment du Saint-Laurent, une société commerciale constituée en 1951, désirait construire une cimenterie dans la municipalité de Villeneuve (devenue la ville de Beauport, un arrondissement de l'actuelle ville de Québec). Afin de lui en donner l'autorisation, une loi spéciale fut adoptée, en 1952, par la législature provinciale. Lorsque s'établit la cimenterie, de nombreux lots sont encore vacants dans le secteur, mais quelques maisons ont déjà été construites sur des terrains contigus à sa propriété. Dès le début de l'exploitation de la Compagnie Ciment du Saint-Laurent, en 1955, des voisins mécontents se plaignent des nuisances résultant des activités de l'usine. Le ministère de l'Environnement intervient à plusieurs reprises dans les années 80 , à l'égard de plaintes formulées par les citoyens relativement à des problèmes de poussière, d'odeurs et de bruits importants ou incessants, selon les témoignages fournis.

La cimenterie produit de son côté plusieurs rapports d'incidents environnementaux. $\mathrm{Au}$ 
début des années 90, Ciment du Saint-Laurent consacre plusieurs millions de dollars à des travaux visant la protection de l'environnement, la majeure partie de cette somme étant affectée à l'installation de nouveaux dépoussiéreurs de fours. En outre, la société accepte de nettoyer à ses frais des maisons et des automobiles salies par des débris ou des poussières provenant de ses opérations.

\section{Alléguant des fautes diverses dans \\ l'exploitation de l'usine ainsi que le caractère anormal ou excessif des troubles de voisinage causés par Ciment du Saint- \\ Laurent, deux citoyens déposent une requête en Cour supérieure.}

Alléguant des fautes diverses dans l'exploitation de l'usine ainsi que le caractère anormal ou excessif des troubles de voisinage causés par Ciment du Saint-Laurent, deux citoyens déposent une requête en Cour supérieure pour obtenir l'autorisation d'exercer un recours collectif au nom des autres résidents demeurant dans des secteurs voisins de la cimenterie. La requête est accueillie par la cour et l'action déposée le $1^{\text {er }}$ août 1994. La compagnie Ciment du SaintLaurent cesse toute exploitation à Beauport en 1997, le conflit avec les voisins se trouvant toujours devant les tribunaux. En 2003, neuf ans après le dépôt de la demande, le tribunal de première instance, en l'occurrence la Cour supérieure du Québec, fait droit au recours collectif sur la base d'un régime de responsabilité sans faute en matière de troubles de voisinage. La Cour supérieure constate qu'il y avait eu respect des normes environnementales applicables et la mise en place des meilleurs équipements antipollution connus. Mais Ciment du Saint-Laurent se trouvait quand même condamnée à verser quelque quinze millions de dollars à deux mille résidents vivant à proximité de la cimenterie. La compagnie porte en appel ce jugement. À l'automne 2006, la Cour d'appel du Québec l'accueille en partie lui faisant droit à l'égard de certains aspects de la détermination des dommages-intérêts qu'elle diminue sensiblement. Elle retient, par contre, la responsabilité civile de la cimenterie sur la base de la faute prouvée, en vertu du régime général de la responsabilité civile, compte tenu de son défaut de respecter certaines normes réglementaires applicables. Le jugement de la Cour d'appel du Québec concernant la cimenterie du SaintLaurent stipule que, dans ce cadre, il incombe à la personne qui poursuit en responsabilité civile un propriétaire pour trouble de voisinage, de prouver la faute du propriétaire, le lien de causalité et le préjudice causé.

\section{Le jugement de la Cour d'appel du Québec concernant la cimenterie du Saint-Laurent stipule que, dans ce cadre, il incombe à la personne qui poursuit en responsabilité civile un propriétaire pour trouble de voisinage, de prouver la faute du propriétaire, le lien de causalité et le préjudice causé.}

La compagnie interjette appel de la décision de la Cour d'appel devant la Cour suprême du Canada. Elle conteste une des conclusions de la Cour d'appel alléguant une responsabilité fondée sur l'existence d'une faute et la méthode d'établissement des dommages. Par ailleurs, deux résidents de Beauport se portent appelants incidents afin de faire reconnaître l'existence d'un régime de responsabilité sans faute fondé sur le caractère excessif des inconvénients du voisinage qu'avait reconnu la Cour supérieure, et pour obtenir le quantum de dommages-intérêts.

Afin de mieux assurer la compréhension de l'affaire et de la décision de la Cour suprême du Canada, il convient de procéder à un bref rappel des notions de responsabilité dans le droit civil québécois. La responsabilité dans le droit civil est particulièrement d'importance pour la bonne gouvernance et pour les activités courantes d'investissement et d'opération des organisations et des entreprises.

La responsabilité dans le droit civil est particulièrement d'importance pour la bonne gouvernance et pour les activités courantes d'investissement et d'opération des organisations et des entreprises. 


\section{LE RÉGIME DE DROIT COMMUN DE LA RESPONSABILITÉ CIVILE EN DROIT QUÉBÉCOIS}

La mise en cause de la responsabilité civile en droit québécois suppose réunis trois ingrédients essentiels : un dommage, une faute et un lien de causalité entre celle-ci et celui-là. En l'absence d'un seul de ces éléments, on ne peut évoquer ou constater la responsabilité civile.

Que veut dire être responsable de ses actes ? Le terme «responsable», du latin respondere, signifie «qui assume les conséquences de ses actes», «être imputable quand aux effets de ses actes », etc. L'assise traditionnelle de la responsabilité civile ${ }^{\mathrm{a}}$, en droit québécois, est fondée sur l'article 1457 du Code civil du Québec qui en énonce les règles générales :

Toute personne a le devoir de respecter les règles de conduite qui, suivant les circonstances, les usages ou la loi, s'imposent à elle, de manière à ne pas causer de préjudice à autrui. (Nous soulignons.)

Elle est, lorsqu'elle est douée de raison et qu'elle manque à ce devoir, responsable du préjudice qu'elle cause par cette faute à autrui et tenue de réparer ce préjudice, qu'il soit corporel, moral ou matériel.

Outre l'obligation de restituer la situation quo ante, c'est-à-dire la condition existant avant le dommage, la mise en cause de la responsabilité civile en droit québécois suppose réunis trois ingrédients essentiels : un dommage, une faute et un lien de causalité entre celle-ci et celui-là. En l'absence d'un seul de ces éléments, on ne peut évoquer ou constater la responsabilité civile. En effet, il importe de souligner que la faute a toujours été une exigence impérative du droit commun de la responsabilité civile. Pour déterminer si une personne a eu un comportement fautif ou non, les tribunaux font appel à certaines normes légales ou réglementaires et ont recours à certains critères comme la diligence et la raisonnabilité.

En tout état de cause, les tribunaux tentent d'apprécier le comportement de la personne, physique ou morale (organisation, entreprise), dont la responsabilité est mise en cause par les faits, de manière à spécifier l'équitable et la nature de la réparation, en tenant compte des circonstances particulières de temps, de lieu, existant au moment où le préjudice est causé.
En tout état de cause, les tribunaux tentent d'apprécier le comportement de la personne, physique ou morale (organisation, entreprise), dont la responsabilité est mise en cause par les faits, de manière à spécifier l'équitable et la nature de la réparation, en tenant compte des circonstances particulières de temps, de lieu, existant au moment où le préjudice est causé. En d'autres termes, pour déterminer s'il y a une faute civile, on évalue ce qu'une personne raisonnable, prudente et diligente (ancien modèle $\mathrm{du}$ 《bon père de famille») aurait fait, placée dans des circonstances comparables. On doit démontrer le caractère socialement répréhensible du comportement de l'auteur. À titre d'exemple, on peut croire respecter les limites de vitesse dans une zone de $50 \mathrm{~km}$, mais si de nombreux écoliers y circulent à l'heure de pointe, il est certainement encore excessif et dangereux pour autrui de rouler à cette vitesse limite ${ }^{b}$. Or, à la lumière des faits mis en preuve dans l'affaire Ciment du Saint-Laurent, il fut jugé qu'aucune faute civile n'avait été commise par la cimenterie, c'est-à-dire en violation de normes légales et réglementaires. Le Règlement sur la qualité de l'atmosphère, le Règlement relatif à l'application de la Loi sur la qualité de l'environnement, le Règlement sur les carrières et sablières et la Loi spéciale de Ciment du Saint-Laurent étaient respectés. Dans son jugement, la Cour suprême estime que Ciment du Saint-Laurent s'était acquittée de son obligation d'employer les meilleurs moyens connus pour éliminer poussières et fumées et que l'équipement utilisé ou installé pour réduire l'émission de contaminants dans l'environnement avait toujours été en bon état de fonctionnement. Mais ce n'était pas suffisant pour « absoudre » Ciment du Saint-Laurent.

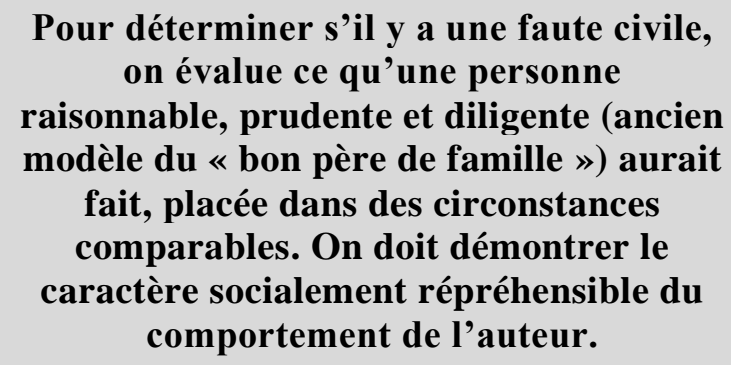
on évalue ce qu'une personne

raisonnable, prudente et diligente (ancien

modèle du « bon père de famille ») aurait fait, placée dans des circonstances comparables. On doit démontrer le caractère socialement répréhensible du comportement de l'auteur. 


\section{LE RÉGIME DE LA RESPONSABILITÉ SANS FAUTE}

Au-delà du régime général de la
responsabilité civile fondé sur la faute, le
plus haut tribunal du pays vient en effet
d'admettre qu'il existe une responsabilité
sans faute dans une situation où des voisins
subiraient des inconvénients (troubles)
anormaux ou excessifs, et ce, sans que le
propriétaire ou le décideur à l'origine de
ces inconvénients ait commis une faute.

Au-delà du régime général de la responsabilité civile fondé sur la faute, le plus haut tribunal du pays vient en effet d'admettre qu'il existe une responsabilité sans faute dans une situation où des voisins subiraient des inconvénients (troubles) anormaux ou excessifs, et ce, sans que le propriétaire ou le décideur à l'origine de ces inconvénients ait commis une faute, contrevenu à une norme établie, commis un abus de droit. Même si cela ne leur fait pas l'économie d'un procès, ce type de responsabilité fait une différence pour les victimes qui n'ont plus la lourde tâche de prouver la «faute » pour obtenir un dédommagement. On n'aura qu'à établir le trouble excessif et le lien de causalité entre le préjudice et le fait générateur du tort. Ce que plusieurs juristes appellent la responsabilité objective fait concentrer l'attention sur le quantum de dédommagement pour la victime, sans égard à une faute, certains juristes y voient en germe le principe de «troubleur-payeur ${ }^{3} »$.

Il importe de rappeler que, si l'application de la responsabilité objective sans faute en matière de troubles de voisinage a été à plusieurs reprises discutée par les tribunaux et par les auteurs de doctrine, le principe n'avait jamais fait l'unanimité au Québec, même s'il s'était à l'occasion manifesté, au Canada et au Québec, dans plusieurs lois et règlements traitant spécifiquement de la protection de l'environnement ${ }^{\mathrm{c}}$. Ce qui a donné ouverture au principe de responsabilité sans faute en situation de troubles de voisinage, en reconnaissance du caractère objectif et excessif des inconvénients subis, c'est l'interprétation qu'a donnée la Cour suprême à un petit article « anodin », peu explicite (et qui aurait pu être rédigé plus clairement), inséré en 1994 dans le Code civil du Québec :

Art. 976. Les voisins doivent accepter les inconvénients normaux du voisinage qui n'excèdent pas les limites de la tolérance qu'ils se doivent, suivant la nature ou la situation de leurs fonds ou suivant les usages locaux.

Lorsqu'on lit attentivement cet article, le législateur ne spécifie pas ce qu'il entend par « voisins », ni la forme que pourra prendre une procédure judiciaire en matière de responsabilité sans faute. Or, la Cour suprême du Canada précise qu'un voisin, c'est un propriétaire, un copropriétaire ou même un locataire qui vit à une certaine proximité géographique du trouble. Il n'est plus nécessaire que les immeubles concernés soient contigus ${ }^{4}$. Par ailleurs, fait d'importance, la cour avalise le recours collectif, qui permet de revendiquer des droits au nom d'un groupe de justiciables, comme une procédure judiciaire admissible aux victimes de désagréments excessifs, mettant ainsi fin à un flottement jurisprudentiel et doctrinal à ce sujet.

Par déduction logique du libellé de l'article 976 du Code civil du Québec, on peut inférer que si les inconvénients ne sont pas excessifs, intolérables, démesurés, allant au-delà de la tolérance normale de la vie de voisinage, il n'y aura pas ouverture au régime de responsabilité sans faute. Un voisin conserve toujours en quelque sorte la liberté de «troubler» dans des « limites raisonnables ». Ce qui fait dire à plusieurs observateurs que la Cour suprême laisse en plan un problème d'importance, car on ne peut fixer aucune balise, critère ou seuil précis permettant de départager des inconvénients acceptables d'inconvénients anormaux justifiant réparation. Pour les organisations, pour les entreprises, leurs activités engendrent de facto une zone d'incertitude. À titre d'exemple, en admettant que cela ne relève pas d'une faute ou d'un abus en soi, le bruit causé par le déneigement de nuit d'un terrain de stationnement adjacent à des résidences privées est-il raisonnable? Le bruit de spectacles musicaux extérieurs organisés durant la période estivale par des organismes sans but lucratif 
demeure-t-il acceptable? Les odeurs de porcheries ou d'épandage de fumier, sont-ils acceptables? L'érection d'une antenne de radio amateur permettant de dispenser un service public (d'urgence ou de sécurité) est-elle une nuisance acceptable pour des voisins, des copropriétaires ou une municipalité? Le juge Gratien Duchesne de la Cour supérieure avait écrit à ce sujet, en 2006, dans l'affaire Daigle c. Caron :

Le même bruit peut être incommodant pour les uns sans l'être pour les autres. C'est une question de seuil de tolérance à ne pas dépasser. Et c'est souvent une question de circonstances les unes plus subjectives que d'autres. (...) Ce seuil de tolérance doit être apprécié en fonction de celui d'autres voisins placés dans les mêmes circonstances : celui de l'être raisonnable (par. 26).

Il importe de rappeler que cette tolérance varie dans le temps, en fonction de l'évolution technologique et des sensibilités. Ce qui était acceptable au $19^{\mathrm{e}}$ ou au $20^{\mathrm{e}}$ siècle ne l'est souvent plus, au début de ce siècle. Les sciences ont fait, ces dernières années, des découvertes importantes sur l'effet nocif de certains produits sur la santé des gens ou encore sur l'influence du bruit excessif en matière de santé physique et mentale de la population ${ }^{5}$. On ne peut exclure que les générations futures développent des perspectives bien différentes des nôtres.
En conséquence, comme le remarque avec justesse Pierre-Claude Lafond, des défenses purement juridiques reposant sur les droits acquis (l'entreprise exerçait ses activités avant le développement résidentiel à proximité) ou sur la responsabilité du dernier arrivé («le dernier arrivé dans le voisinage doit se plier à la loi du premier arrivant $\left.{ }^{6} \gg\right)$ ne peuvent être reçues, car elles jettent un voile sur la vraie dimension sociale des troubles de voisinage. Un « voisin» doit assumer la nécessité d'être solidaire de la société et du monde qui l'entoure, qu'il soit arrivé dans le voisinage en premier ou pas.

Ainsi, le droit de propriété et la liberté individuelle perdent le caractère en apparence absolu qu'ils semblaient avoir dans un contexte idéologique ou institutionnel néolibéral (dominé par le concept de libre marché), et se voient assigner à nouveau des limites, dans l'esprit de la promotion de la vie civique et d'un véritable développement c'est-à-dire, des transformations psychosociales et institutionnelles qui favorisent l'expansion et la croissance, la réduction des inégalités sociales, la préservation de l'environnement, etc.

Un « voisin » doit assumer la nécessité d'être solidaire de la société et du monde qui l'entoure, qu'il soit arrivé dans le voisinage en premier ou pas.

\section{LES CONSÉQUENCES DU RÉGIME DE LA RESPONSABILITÉ SANS FAUTE POUR LA GESTION DU RISQUE ET DE LA COMPLEXITÉ}

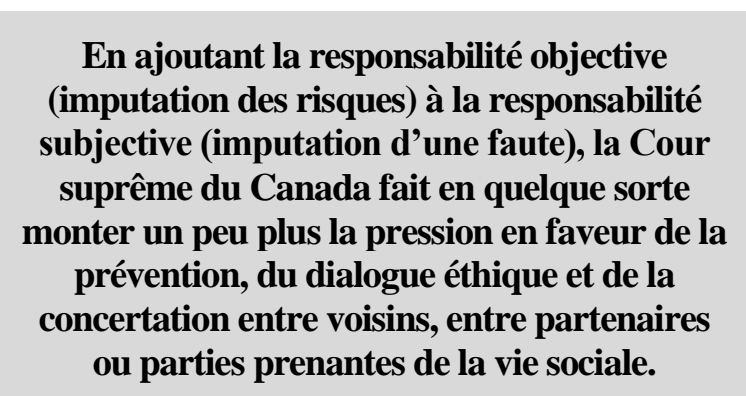

En ajoutant la responsabilité objective (imputation des risques) à la responsabilité subjective (imputation d'une faute), la Cour suprême du Canada fait en quelque sorte monter un peu plus la pression en faveur de la prévention, du dialogue éthique et de la concertation entre voisins, entre partenaires ou parties prenantes de la vie sociale. Cette nouvelle balise d'ordre juridique augmente inévitablement le niveau de complexité de la prise de décision des gestionnaires des organisations publiques et privées.

Qu'est qui est tolérable? Qu'est-ce qui ne l'est pas? On ne trouvera pas de balises juridiques claires à cet effet dans la loi; chaque cas étant un cas d'espèce devant faire l'objet d'une implication active des parties en cause. Le gestionnaire d'une organisation est alors directement appelé à participer à la définition du devenir de l'organisation ou de l'entreprise, et 
surtout à ne pas négliger de considérer la contribution des parties prenantes, actuelles ou futures, touchées par ses décisions ${ }^{7}$. Le gestionnaire, voire l'organisation elle-même, se voient en ce sens reconnus dans leur condition de personnes morales et de parties prenantes à la redéfinition incessante d'un contrat moral avec les autres parties prenantes (citoyens, municipalités, État), c'est-à-dire la communauté dont ils sont membres en permanence.

Ce contrat moral peut être décrit comme un ensemble de règles et de comportements assurant aux parties prenantes que le lien de confiance se maintient, contribuant de ce fait à préserver les avantages mutuels d'un système de coopération. Ainsi, une organisation économique pourra bénéficier des avantages de la complexité sans se perdre au jeu du prisonnier dans lequel on cherche à se donner un avantage au détriment de ses partenaires. Une structure sociale saine et une bonne gouvernance permettent de redéfinir régulièrement les modalités de la confiance et de la coopération entre les associés de fait.

En raison de leur évolution constante, ces contrats moraux ne sauraient être définis uniquement par une structure de normes juridiques. Cette structure doit cependant en prescrire les contours, afin d'inciter les partenaires sociaux à ne jamais cesser de redéfinir les modalités de leur jeu social, de façon à le préserver, compte tenu des changements qu'entraîne la compétition survenant de l'extérieur, comme de l'intérieur, par suite de changements dans la technologie et la productivité du travail.

Le jugement de la Cour suprême rappelle aux partenaires sociaux leur responsabilité de combler, dans les circonstances de leur vie commune, le vide laissé par la loi par le biais d'une négociation régulière de leur contrat moral de coopération et de bon voisinage, de manière à réaliser plus de justice dans les rapports sociaux. On n'espère pas atteindre la perfection, mais au moins à réduire les injustices les plus graves (les troubles anormaux ou excessifs) qui font plus facilement un large consensus.

Sur le plan concret, la solidarité devient un principe de surordination généralisé du comportement, fondant une gouvernance commune des choses communes, appelant à la mise en œuvre de formes diverses de participation responsable, replaçant ainsi le dialogue au centre des pratiques de gestion. À l'issue d'exercices non truqués, authentiques d'information, de consultation et d'entente, on connaitrait des périodes de tolérance ou d'adaptation mutuelle. La bonne gouvernance privée ou publique procurerait alors un modus vivendi allant au-delà des conditions de conformité à une démarche préétablie, à une procédure ou à un ensemble déjà déterminé de règles. Les auteurs David Duchamp et Jacky Koehl confirment que,

le sentiment indéniable d'intensification de la complexité managériale qui résulte de l'émergence des nouveaux paradigmes (...) (développement durable, RSE...) nous semble comporter un avantage non négligeable: la douce illusion des outils, des recettes-miracles s'estompe ${ }^{8}$.

\section{La solidarité devient un principe de surordination généralisé du comportement, fondant une gouvernance commune des choses communes, appelant à la mise en ouvre de formes diverses de participation responsable, replaçant ainsi le dialogue au centre des pratiques de gestion.}

Cette négociation entre les partenaires du bon voisinage implique de s'engager dans un cycle décisionnel permanent. La fonction essentielle d'un tel cycle décisionnel consiste à recadrer les perceptions et les schèmes de pensée des acteurs, sur la base des contraintes et des opportunités de la situation, sur la base de modifications dans les échelles de valeurs et de nouvelles perspectives apportées par le progrès des sciences et techniques.

Le psychosociologue Kurt Lewin a montré que ce recadrage va toucher tous les partenaires, y compris les scientifiques, dans leur manière de voir la réalité, de façon à créer une situation améliorée par rapport à celle de départ, au statu quo ante. Cette démarche, qui se trouve bien dans l'esprit de la gouvernance, peut alors effectuer le travail d'ajustement mutuel et de bon voisinage prescrit expressément, mais de façon ouverte, par la loi. 


\section{LE NOUVEAU RÉGIME JURIDIQUE EN MATIÈRE DE TROUBLE DE VOISINAGE ET LE DÉVELOPPEMENT DURABLE}

Une règle généralement reconnue en interprétation des lois présuppose la rationalité du législateur. On suppose qu'il règne, entre les divers textes législatifs adoptés par une même autorité, la même harmonie que celle que l'on trouve entre les divers éléments d'une loi.

Une règle généralement reconnue en interprétation des lois présuppose la rationalité du législateur. On suppose qu'il règne, entre les divers textes législatifs adoptés par une même autorité, la même harmonie que celle que l'on trouve entre les divers éléments d'une loi. Comme le rappelle Pierre-André Côté, «l'ensemble des lois est censé former un tout cohérent $^{9}$ ». On peut ainsi faire un rapprochement intéressant entre le régime de responsabilité objective sans faute en matière de troubles de voisinage et l'esprit du développement durable. Cela forme, à notre avis, un tout rationnel indissociable. Et de fait, la Cour suprême du Canada a d'ailleurs fait ce rapprochement dans l'affaire Ciment du St-Laurent en mentionnant que le régime de la responsabilité civile fondé sur l'existence de troubles de voisinage anormaux «s'accorde avec des considérations de politique générale, tels que l'objectif de protection de l'environnement et l'application du principe du pollueur-payeur $»$. Selon la Cour suprême du Canada, la responsabilité sans faute renforce spécifiquement l'application du principe de pollueur-payeur qu'elle avait d'ailleurs examiné dans l'affaire Cie pétrolière Impériale ltée c. Québec (Ministre de l'Environnement), [2003] 2 R.C.S. 624.

Dans une optique de développement durable, ce principe impose aux pollueurs la responsabilité de corriger les situations de contamination dont ils sont responsables et d'assumer directement et immédiatement les coûts de la pollution. Il cherche en même temps à rendre les auteurs de celle-ci plus soucieux des impératifs de la préservation des écosystèmes, dans le cours de leurs activités économiques. [par. 24]

Il convient de rappeler que le législateur québécois a inscrit le principe de pollueur-payeur, mais aussi celui de la participation citoyenne lorsqu'il adopte en avril 2006 la Loi sur le développement durable L.R.Q. c. D-8.1.1. Cette loi affirme avec force sa volonté de restructurer la gouvernance et, par voie de conséquence, le cadre de gestion de l'administration publique québécoise. L'exercice des pouvoirs et des responsabilités des ministères, organismes et entreprises d'État (et, sur décret, les universités, les municipalités et les hôpitaux) doit désormais s'inscrire dans la recherche d'« un développement qui répond aux besoins du présent sans compromettre la capacité des générations futures à répondre aux leurs ${ }^{\mathrm{d}} \gg$. Le concept de développement durable s'appuie essentiellement sur une vision à long terme qui prend en compte «le caractère indissociable des dimensions environnementales, sociales et économiques des activités de développement ${ }^{10} »$. Ainsi, la participation citoyenne au développement durable apparait comme un principe de base. Le développement durable implique en son principe même des changements de comportements, la sensibilisation de chacun et la participation de tous au processus de décision à travers différents mécanismes de démocratie participative $^{11}$.

Dans un tel contexte, l'État devient une organisation plus réflexive et elle-même en développement, plus en phase avec les mouvements sociaux. On renonce à un modèle tutélaire de résolution de problèmes, pour convier les acteurs sociaux à une forme de gouvernance participative. Les organisations privées sont visées indirectement par cette loi, car elles se voient accorder chaque année, par les organismes gouvernementaux et paragouvernementaux, des contrats de plusieurs milliards de dollars. Elles sont donc tenues de s'inscrire dans un cadre de gestion impliquant les autres parties prenantes, y compris celles dites $\ll$ secondaires ${ }^{12} »$.

Le développement durable implique en son principe même des changements de comportements, la sensibilisation de chacun et la participation de tous au processus de décision à travers différents mécanismes de démocratie participative. Dans un tel contexte, l'État devient une organisation plus réflexive et elle-même en développement, plus en phase avec les mouvements sociaux. 


\section{CONCLUSION}

\section{Les décideurs privés doivent s'assurer, de façon préventive, plutôt que réactive, de mettre en place toutes les conditions pour que leurs opérations « dans le voisinage » respectent les droits d'autrui et ce, avant même que les troubles ne deviennent excessifs.}

La décision de la Cour suprême du Canada dans l'affaire Ciment du Saint-Laurent renforce, s'il en était encore besoin, le sentiment que les décideurs privés doivent veiller à bien assumer leur responsabilité en matière de gouvernance. Les décideurs privés doivent s'assurer, de façon préventive, plutôt que réactive, de mettre en place toutes les conditions pour que leurs opérations «dans le voisinage» respectent les droits d'autrui et ce, avant même que les troubles ne deviennent excessifs. Cette gouvernance privée devra être tout à fait réflexive, en relation avec les autres parties prenantes potentiellement touchées. Cela va requérir un accroissement des qualités des gestionnaires, une adaptation des programmes de formation des décideurs et de leurs collaborateurs. Il s'agit ni plus ni moins de réinventer la façon de faire la gestion du risque au quotidien, la façon d'aborder le cycle décisionnel. Les organisations devront évaluer concrètement leur responsabilité sociale. Nous sommes tous en fait conviés à rétablir la primauté du lien social, les conditions du mutualisme, la solidarité devant prévaloir sur les horizons forcément bornés des rapports sociaux mercantiles.

\section{BIBLIOGRAPHIE}

${ }^{1}$ Ciment du Saint-Laurent inc. c. Barrette, [2008] 3 R.C.S. 392.

${ }^{2}$ À ce sujet, on écoutera : Radio-Canada, « La sourde d'oreille », Enquêtes, 19 novembre 2009. On pourra consulter le reportage à l'adresse suivante : http://www.radio-canada.ca/emissions/enquete/20092010/Reportage.asp?idDoc $=106561 ;$ Marc SaintPierre, "Ciment Saint-Laurent inspire les AntiRabaska », Le Soleil, le 3 décembre 2008. Logiquement le gouvernement ne devrait donc pas tarder à modifier la Loi sur les véhicules hors routes, L.R.Q. c. V-8.1, qui interdit, depuis décembre 2004, tout recours intenté sur la base d'inconvénients de voisinage ou de préjudice lié aux bruits, aux odeurs ou à d'autres contaminants du fait de l'utilisation de motoneiges ou de véhicules tout-terrain.

${ }^{3}$ Arbour, M.-È. et Racine, V. (2009). «Itinéraires du trouble de voisinage dans l'espace normatif », Cahiers de droit, vol. 50, $\mathrm{n}^{\mathrm{o}} 2,327,358$.

${ }^{4}$ Lafond, P.-C. (2009) « L'heureuse alliance des troubles de voisinage et du recours collectif : portée et effets de l'arrêt Ciment du Saint-Laurent ", Revue du Barreau, vol. 68, 385, 443.

${ }^{5}$ Bouchard, M. et Smargiassi, A. (2008). Estimation des impacts sanitaires de la pollution atmosphérique au Québec : essai d'utilisation du Air Quality Benefits Assessment Tool (AQBAT), Institut national de santé publique du Québec; Leroux, T. et Gagné, J.P. (2007). Évaluation des impacts sur la santé des populations vivant à proximité des parcs éoliens, École d'orthophonie et d'audiologie, Université de Montréal. On trouvera au Québec de nombreux mouvements citoyens : Le regroupement québécois contre le bruit -excessif (RQCB) http://www.rqcb.ca/ fr/accueil.php;

${ }^{6}$ Lafond, P.-C. (2009). " L'heureuse alliance des troubles de voisinage et du recours collectif : portée et effets de l'arrêt Ciment du Saint-Laurent », Revue du Barreau, vol. 68, 385, 442.

${ }^{7}$ Senge, P. M. (2000). La cinquième discipline : le guide de terrain : stratégies et outils pour construire une organisation apprenante, Paris, Frest.

${ }^{8}$ Duchamp, D. et Koehl, J. (2008). « L'éthique de la discussion au service de la performance organisationnelle. Pour l'avènement de la compétence délibérative dans le profil managérial », Revue internationale de psychologie, no 3, vol. XIV, p. 201.

${ }^{9}$ Côté, P.-A., Beaulac, S. et Devinat, M. (2009). Interprétation des lois, $4^{\mathrm{e}}$ éd., Montréal, Les éditions Thémis, p. 395.

${ }^{10}$ Bourque, D. (2009) « La formation en gestion et le développement durable ", Organisations et territoires, vol 18, $\mathrm{n}^{\mathrm{0}} 1,71-76$.

${ }^{11}$ On lira l'Orientation 8 « Favoriser la participation à la vie collective » de la Stratégie gouvernementale de développement durable 2008-2013, Québec, décembre 2007, p. 53 et 54.

${ }^{12}$ Dion, M. et Fortier, M. (2011). Les enjeux éthiques de l'entreprise, Éditions du Renouveau Pédagogique Inc., Saint-Laurent, 109-125.

\section{Notes}

${ }^{a}$ Il faut faire la différence entre la responsabilité civile et la responsabilité pénale. La responsabilité civile est l'obligation de réparer le dommage que l'on cause à autrui. Dans les cas les plus graves, la responsabilité pénale renvoie à la punition ou à la sanction d'un individu qui commet une infraction (contravention, délit, crime). 
${ }^{\mathrm{b}}$ En droit civil, une personne n'est pas admise à faire un mauvais usage des droits dont elle est titulaire, en d'autres termes à en abuser. Elle est tenue de les exercer dans le respect de certaines règles, notamment celles de la bonne foi et de l'obligation de ne pas priver autrui de la jouissance de ses droits. Ainsi, auraient un comportement fautif susceptible d'engager leur responsabilité civile aussi bien celui qui exerce un droit en vue de nuire à autrui que celui qui l'exerce de manière excessive et déraisonnable. Dans le premier cas, la preuve de l'intention malicieuse (intention de nuire) est requise; elle peut être inférée des faits démontrés. À titre d'exemple, un propriétaire, croyant que ses voisins ne bénéficiaient d'aucun droit de passage sur son terrain, avait abusé de son droit en bloquant le passage sans attendre qu'un tribunal ne se soit prononcé sur l'existence de la servitude. Dans un autre cas de figure dans la jurisprudence, la preuve de ce que constitue un comportement excessif et déraisonnable résultait d'une appréciation objective de la conduite de la personne, soit de l'observation des torts causés; il n'était aucunement nécessaire de prouver une intention malicieuse expresse pour établir l'abus de droit. Enfin, dernier exemple, on peut considérer que constituerait un abus de droit la construction d'une haute clôture ayant pour effet de priver un voisin de la majeure partie de sa vue sur un fleuve ou des montagnes. ${ }^{c}$ Avant le jugement de la Cour suprême du Canada dans l'affaire Ciment du Saint-Laurent, le régime de responsabilité objective (ou sans faute) s'était à l'occasion manifesté, au Canada et au Québec, dans plusieurs lois et règlements traitant spécifiquement de la protection de l'environnement, par la voie du principe de «pollueurpayeur ». Ce principe, on le rappelle, a été adopté sur le plan international par l'OCDE en 1972. Il représente une conception critique d'une certaine vision de la sphère économique, qui implique que le pollueur prenne en compte (internalise) les coûts de l'utilisation ou de la détérioration des ressources environnementales, et cesse de pratiquer ce que l'on appelle l'externalisation des coûts, c'est-à-dire le renvoi des coûts sur la société et l'environnement, tout en conservant les bénéfices de l'opération (on parle alors de socialisation des coûts et de privatisation des profits).

${ }^{\mathrm{d}} \mathrm{La}$ Loi sur le développement durable a aussi introduit dans la Charte [québécoise] des droits et libertés de la personne, le droit de chacun « de vivre dans un environnement sain et respectueux de la biodiversité» (art. 46.1). En donnant une valeur quasi constitutionnelle au « droit à un environnement sain », la protection de l'environnement est devenue en quelque sorte une valeur fondamentale de la société québécoise et un impératif collectif, c'est-à-dire une valeur-pivot affectant toutes les variables du développement. 